\title{
Identification of genomic aberrations in hemangioblastoma by droplet digital PCR and SNP microarray highlights novel candidate genes and pathways for pathogenesis
}

\author{
Ruty Mehrian-Shai ${ }^{{ }^{*}}$, Michal Yalon ${ }^{1 \dagger}$, Itai Moshe ${ }^{1}$, Iris Barshack ${ }^{2,3}$, Dvorah Nass ${ }^{3}$, Jasmine Jacob ${ }^{1}$, Chen Dor ${ }^{1}$, \\ Juergen K. V. Reichardt ${ }^{4}$, Shlomi Constantini ${ }^{2,5+}$ and Amos Toren ${ }^{1,2 \dagger}$
}

\begin{abstract}
Background: The genetic mechanisms underlying hemangioblastoma development are still largely unknown. We used high-resolution single nucleotide polymorphism microarrays and droplet digital PCR analysis to detect copy number variations (CNVs) in total of 45 hemangioblastoma tumors.

Results: We identified 94 CNVs with a median of 18 CNVs per sample. The most frequently gained regions were on chromosomes 1 (p36.32) and 7 (p11.2). These regions contain the EGFR and PRDM16 genes. Recurrent losses were located at chromosome 12 (q24.13), which includes the gene PTPN11.

Conclusions: Our findings provide the first high-resolution genome-wide view of chromosomal changes in hemangioblastoma and identify 23 candidate genes: EGFR, PRDM16, PTPN11, HOXD11, HOXD13, FLT3, PTCH, FGFR1, FOXP1, GPC3, HOXC13, HOXC11, MKL1, CHEK2, IRF4, GPHN, IKZF1, RB1, HOXA9, and micro RNA, such as hsa-mir-196a-2 for hemangioblastoma pathogenesis. Furthermore, our data implicate that cell proliferation and angiogenesis promoting pathways may be involved in the molecular pathogenesis of hemangioblastoma.
\end{abstract}

Keywords: Hemangioblastoma, CGH, digital PCR, cancer

\section{Background}

Hemangioblastomas $(\mathrm{HB})$ are highly vascular tumors, which account for approximately $3 \%$ of all tumors of the central nervous system (CNS) [1]. It occurs in a subset of CNS locations, including the cerebellum (37\%), brainstem (10\%), and spinal cord (50\%) [1]. They are classed as grade one tumors under the World Health Organization's classification system. While most of these tumors are low grade and benign, some hemangioblastomas can present aggressive and occasionally malignant behavior. Hemangioblastomas occur as sporadic tumors $(75 \%)$ or as a

\footnotetext{
*Correspondence: ruty.shai@sheba.health.gov.il

${ }^{\dagger}$ Equal contributors

${ }^{1}$ Pediatric Hemato-Oncology, Edmond and Lilly Safra Children's Hospital and Cancer Research Center, Sheba Medical Center, Tel Hashomer affiliated to the Sackler School of Medicine, Tel-Aviv University, Tel Aviv, Israel Full list of author information is available at the end of the article
}

manifestation of an autosomal dominantly inherited disorder, von Hippel-Lindau (VHL) disease (25 \%) [2].

VHL hemangioblastomas are most commonly caused by germline exon deletions or truncating mutations [3] of the Von Hippel-Lindau (VHL) tumor-suppressor gene. The VHL protein, which is the critical part of a ubiquitin ligase protein complex that binds to the hypoxia-inducing factors HIF-1 and HIF-2 transcription factors and targets them for ubiquitination and proteosomal degradation. Dysregulation of this VHL-associated function causes increased expression of a variety of growth factors, including erythropoietin, PDGF, VEGF and TGF. Upregulation of these factors may lead to angiogenesis and tumorigenesis. Additional mechanisms of tumorigenesis have been described outside of the HIF pathway, including alterations in microtubule binding and stabilization, abnormal 
extracellular matrix composition as well as apoptosis and transcription regulation [4]. For most VHL disease related hemangioblastomas, the inactivation or loss of both alleles of the VHL gene is required. In addition to the phenotypic variability associated with allelic heterogeneity, genetic modifiers may influence the phenotypic expression of VHL disease. Allelic variants in the CCND1, MMP1 and $M M P 3$ genes have been reported to influence hemangioblastoma development [5]. This reiterates the need for elucidating other genetic alterations specific for hemangioblastoma beside the hits of $V H L$ gene. Moreover, in a subset of tumors including mostly sporadic hemangioblastomas, the genetic pathways involved in tumorigenesis have not been defined yet [6].

Copy number variants (CNVs) are alterations of DNA sections in result of genomic deletions (fewer than the normal number) or duplications (more than the normal number) on certain chromosomes and are common to many human cancers. Comparative genomic hybridization (CGH) by single nucleotide polymorphism (SNP) arrays is a cutting edge technology that allows characterization of CNVs. SNP array karyotyping provides genome-wide assessment of copy number and loss of heterozygosity (LOH) in one assay. SNP array platforms, such as Affymetrix SNP 6.0 (Affymetrix, Santa Clara, CA, USA), often identify amplifications/deletions at a single gene level, which could not have been accomplished by previous methods. Thus, modern SNP arrays offer a powerful method for the discovery of oncogene and tumor suppressor gene involvement in tumors, as well as for improved cancer classification [7].

In contrast to surveillance of genome wide alterations by CGH arrays it is possible to directly quantify the absolute copy number of specific DNA loci by Droplet Digital PCR (ddPCR). In ddPCR, target sequences are amplified by PCR and the reaction products are partitioned into droplets and amplified to endpoint with TaqMan probes as in qPCR, then their concentrations are determined based on the number of fluorescently positive and negative droplets in a sample well. The absolute number of target and reference DNA molecules is calculated and provides the target copy number variation (CNV) [8].

In the present study we used high-resolution SNP arrays for the first time to genome wide analysis of aberrations in hemangioblastomas aiming at the identification of novel pathogenetic mechanisms and possible targets for rational therapy. We validated the main reoccurring genetic changes by ddPCR highly precise quantification.

\section{Methods}

\section{Study population}

A total of 44 hemangioblastoma samples were used for the present study. Thirteen frozen samples obtained from The Sourasky Medical Center, Tel Aviv, Israel were used for the CGH analysis. Additional 32 formalin fixed paraffin embedded (FFPE) samples from Sheba Medical Center, Tel Hashomer, Israel were used as validation group. The study was approved by the ethical review boards of both Sheba and Tel Aviv Sourasky Medical Centers and was consistent with the declaration of Helsinki including informed consents. Clinical parameters, such as sex, age at diagnosis, and pathologic classification were collected from patient records. Clinical information of the patient's cohort is outlined in Table 1 .

\section{CGH analysis}

DNA was purified from frozen tissues using DNeasy (Qiagen Inc., Valencia, CA). One sample of pooled normal genomic DNA, provided by Affymetrix, was used as experimental positive control. 250 ng of genomic DNA was digested with $N s p$ I (New England Biolabs, Inc) and then ligated to Nsp adaptors. The adaptor-ligated DNA fragments were amplified, fragmented using DNase I, end labelled with a biotinylated nucleotide, and hybridized to a human cytoscan HD array (Affymetrix) at $50^{\circ}$ $\mathrm{C}$ for $17 \mathrm{~h}$. After hybridization, the arrays were washed, stained, and finally scanned with a GeneChip scanner 3000 (Affymetrix). All procedures were performed according to the manufacturer's protocols. Array experiments were performed using the high-resolution Affymetrix CytoScan HD microarray (Affymetrix, Inc, Santa Clara, CA) containing 2,696,550 markers of which $1,953,246$ are non-polymorphic markers and 750,000 SNPs with over $99 \%$ accuracy to detect accurate breakpoint estimation as well as loss of heterozygosity (LOH) determination. This chip covers 340 International Standards for Cytogenomic Arrays (ISCA) constitutional genes, 526 cancer genes (99.6\%) and 36,121 RefSeq genes. The chip uses marker intervals of 25 markers / $100 \mathrm{~kb}$. Analysis of CEL files from the Affymetrix CytoScan HD Array or Cytogenetics Whole-Genome 2.7 M Array was done with the Chromosome Analysis Suite (ChAS) software for cytogenetic analysis. Signal processing was done by Signal Covariate Adjustment, Fragment Correction, Dual Quantile Normalization and PLIER signal summarization. Dual Quantile Normalization was done to equalize each array's intensity distribution copy number and SNP probes separately. For SNP

Table 1 Cohort characteristics

\begin{tabular}{lll}
\hline Characteristic & Frozen & Paraffin \\
\hline Average age & 48.5 & 53.5 \\
Median age & 51 & 53 \\
Spinal samples & 4 & 3 \\
Brain samples & 8 & 29 \\
Total number & 12 & 32 \\
\hline
\end{tabular}


markers, multiple probes for each allele were summarized to single values. Copy number $(\mathrm{CN})$ was calculated by hidden Markov model copy number segments after $\log 2$ calculation, high pass filter image correction, $\log 2$ ratio covariate adjustment and systematic residual variables removal. The baseline for $\mathrm{CN}=2$ (normal autosomal copy number state) was established and used by the analysis software by Affymetrix company using a set of 380 phenotypically normal individuals named as reference. The reference sample includes 186 females and 194 male. For chromosome $\mathrm{X}$, only females were used and for chromosome $\mathrm{Y}$ only males were used. Log2 ratios for each marker are calculated relative to the reference signal profile. Results of the summarized Data (CYCHP files) were viewed as chromosomal aberrations in table and graphical formats. We also added visual inspection of probe performance for altered segments. Reference intensity intended to represent the copy normal state (typically 2). Log ratios above 0 mean $\mathrm{CN}$ gain, $\log$ ratios below 0 mean $\mathrm{CN}$ loss and $\log$ ratios around 0 represent no change. Abnormal DNA copy numbers are identified automatically using 25 markers for loss/ 50 markers for gains.

\section{VHL sequencing}

To screen the $V H L$ gene for mutations in our cohort, we performed direct sequencing of the coding region. Exons 1,2 and 3 of the $V H L$ gene and their immediately flanking sequences were amplified by PCR as described previously [9]. The PCR amplification products were purified by using the QIAquick PCR Purification Kit (Qiagen), according to the manufacturer's instructions. The amplification primers were used as primers in the sequencing reactions, except for exon 1 , for which we designed a new cycle sequencing primer (5'CGAAGATACGGAGG TCGA3'). Cycle sequencing was performed using the ABI PRISM Big Dye Terminator Cycle Sequencing Ready reaction kit (Applied Biosystems, Foster City, CA, USA), followed by isopropanol precipitation. The fragments were sequenced by automated sequencing analysis on an ABI Prism 377 sequencer (Applied Biosystems).

\section{Droplet digital PCR}

Copy Number validation was done on all samples, frozen and paraffin embedded, hemangioblastoma biopsies. Genomic DNA was purified using QIAamp DNA mini (Qiagen). Copy number variation (CNV) test was performed by droplet digital PCR (ddPCR) as previously described [10]. In short, 16 ng of genomic DNA samples were added to 2xddPCR supermix (Bio-Rad) with final concentration of $500 \mathrm{nM}$ of each primer and $250 \mathrm{nM}$ probe in duplex of the tested gene and RNaseP. $\mathrm{RNaseP}$ served as a $\mathrm{CNV}=2$ reference gene. Probes for the tested genes contained a FAM reporter and RNaseP contained HEX. The genomic DNA and PCR reaction mixtures were partitioned into an emulsion of approximately 20,000 droplets using the QX100 droplet generator (Bio-Rad, USA). The droplets were transferred to a 96-well PCR plate, heat sealed, and placed in a conventional thermal cycler. Thermal cycling conditions were: $95 \mathrm{oC}$ for $10 \mathrm{~min}$ followed by 40 cycles of $94 \mathrm{oC}$ for $30 \mathrm{~s}$, $60 \mathrm{oC}$ for $60 \mathrm{~s}$ and one cycle of $98 \mathrm{oC}$ for $10 \mathrm{~min}$ and finally $4 \mathrm{oC}$ hold. Following PCR, the plates were loaded into QX100 droplet reader (Bio-Rad) and the CNV value was calculated using Quantasoft software (Bio-Rad, USA). Primers and probes for selected areas enlisted in Table 2 were designed using Primer Express software (PE Corp, USA) and specificity was verified using NCBI BLAST online tool (National Library of Medicine, USA). RNaseP primers and probes were obtained from Bio-Rad.

\section{Results}

SNP array profiling identifies recurrent CNVs

The systematic genome-wide gain and loss segments based CNVs data detected by SNP profiling in hemagioblastoma is detailed in the karyoview (Fig. 1). We identified $94 \mathrm{CNVs}$ with a median of $18 \mathrm{CNVs}$ per sample. Twenty-three of them involved noncoding regions in centromeres that are known to harbor spurious CNVs, most of them were less than $100 \mathrm{~Kb}$ long, and 56 were found in at least two samples. The most frequently gained regions were on chromosomes 1 (p36.32) and 7 (p11.2). These regions contain the PRDM16 and EGFR genes, respectively. Recurrent losses were located at chromosome 12 (q24.13) which includes the PTPN11 gene. Pathway analysis revealed that EGFR, Notch and HedgeHog signaling were the most frequently altered pathways promoting angiogenesis and proliferation. Mir 551a (part of PRDM16) gain was detected in seven samples, miR-196a-2 gain was observed in five samples and miR-196b gain was detected in four samples. The list of recurrent CNVs found in at least five specimens (Fisher exact test $p$-value $<$ 0.05), including type of alteration, involved chromosome, cytobands, and overlapping genes/miRNAs according to the RefSeq database is provided in Table 3. Two samples had $\mathrm{LOH}$ affecting the CHECK2 region (Fig. 2).

\section{VHL status}

We determined the VHL mutation status of our discovery cohort by both DNA sequencing and results from the CGH arrays. In two of the thirteen frozen samples (samples 7 and 10) VHL deletions were detected by our CGH analyses. The deletion in sample 7 encompassed $332 \mathrm{~kb}$ with 564 markers. The deletion in sample 10 was shorter $(135 \mathrm{~kb})$ with 344 markers. Figure 3 illustrates the smooth signal obtained in chromosome band 
Table 2 Digital PCR primers and probes for selected areas

\begin{tabular}{|c|c|c|c|}
\hline Gene & Oligonucleotides & Sequence $\left(5^{\prime}-3^{\prime}\right)$ & reporter \\
\hline \multirow[t]{3}{*}{ PTPN11 } & Forward primer & TTAGAGACAGGGTCCCACTCTTG & - \\
\hline & Reverse primer & GCTTGAGGATGCAGTAAGCTATGA & - \\
\hline & Probe & CCTGGCTGGAGTGCAGTGGCGT & FAM \\
\hline \multirow[t]{3}{*}{ CHECK2 } & Forward primer & CATIITCTCTTAGTATCTITCTGGGAAT & - \\
\hline & Reverse primer & CATTTCTGAGCCCAGCAATACA & - \\
\hline & Probe & TCACAATCCAGGGCTACAGTAAGACCCATG & FAM \\
\hline \multirow[t]{3}{*}{ PTCH1 } & Forward primer & GCGTGCGAAGGTGGAGACT & - \\
\hline & Reverse primer & TCATTGGCCTCCCACTTGA & - \\
\hline & Probe & TGTCTTCTCCCCCATGTCGG & FAM \\
\hline \multirow[t]{3}{*}{ EGFR } & Forward primer & AGGAGGAACAACGTGGAGACA & - \\
\hline & Reverse primer & GAGACACCGGAGCCACAGA & - \\
\hline & Probe & CCCAGAGGTGGAACGTTGGCCC & FAM \\
\hline \multirow[t]{3}{*}{ RNaseP } & Forward primer & GATTTGGACCTGCGAGCG & - \\
\hline & Reverse primer & GCGGCTGTCTCCACAAGT & - \\
\hline & Probe & CTGACCTGAAGGCTCT & Hex \\
\hline
\end{tabular}

3p25.3 for these samples. Sequencing based mutational analysis of $V H L$ did not reveal any additional mutations. Samples 7 and 10 incurred CNVs similarly to the other samples. The average number of CMVs in the other samples was seven. Sample 7 had 14 of the common CNVs thus had more than the average CNVs but sample 10 had less CNVs (six) which is similar to the average.
CNV validation by digital droplet PCR

We validated our findings in a subset of four genes (EGFR, CHECK2, PTCH1 and PTPN11) in the discovery cohort used for the array $\mathrm{CGH}$ and in an additional independent set of 32 FFPE specimens, using copy variation detection by digital droplet PCR (ddPCR) analysis. Samples were partitioned into thousands of nanolitersized droplets; single template molecules were amplified

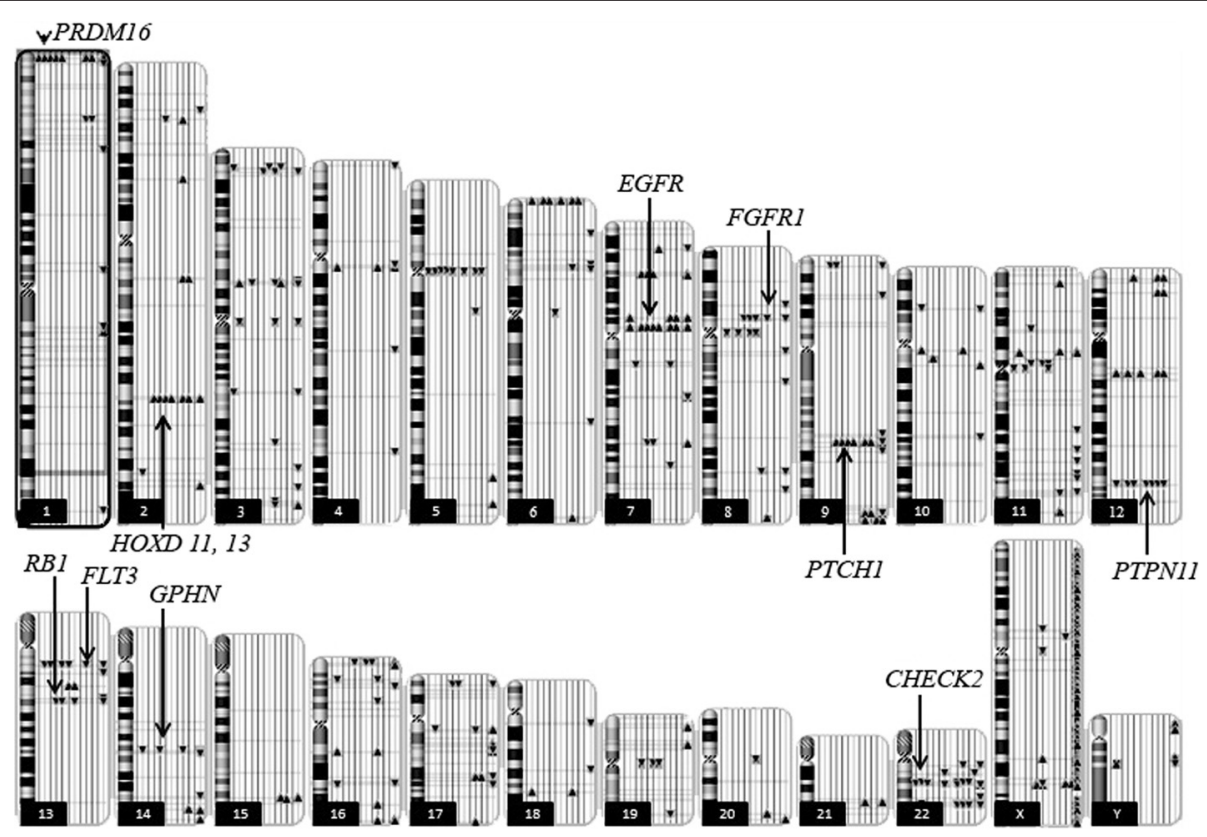

Fig. 1 Ideogram illustrating genome-wide distribution of DNA amplifications and deletions (copy number variation) on each chromosome. Each line next to the chromosome represents one sample. Gains in copy number are represented by triangles pointing upwards and losses in copy number are represented by triangles pointing downwards with indication of gene in that region. Chromosome numbers are indicated in boxes 
Table 3 Frequent chromosome losses and gains involving single genes

\begin{tabular}{|c|c|c|c|c|c|c|}
\hline Number of samples & Type & Chromo some & Band & Gene & miRNA & GV \\
\hline 7 & Gain & 7 & p11.2 & EGFR & & - \\
\hline 7 & Gain & 1 & p36.32 & PRDM16 & hsa-mir-551ahsa-mir-551a & $v$ \\
\hline 7 & Loss & 12 & $\mathrm{q} 24.13$ & PTPN11 & & - \\
\hline 7 & Gain & 2 & q31.1 & $\begin{array}{l}\text { HOXD11, HOXD13 HOHOHOXD13 } \\
\text { HOXD11HOXD13 }\end{array}$ & & $v$ \\
\hline 6 & Loss & 13 & $q 12.2$ & FLT3 & & - \\
\hline 6 & Gain & 9 & $\mathrm{q} 22.32$ & PTCH & & $v$ \\
\hline 5 & Loss & 8 & p11.22 & FGFR1 & & - \\
\hline 5 & Loss & 3 & $\mathrm{p} 13$ & FOXP1 & & - \\
\hline 5 & Gain & $x$ & q26.2 & GPC3 & & - \\
\hline 5 & Gain & 12 & q13.13 & HOXC13, HOXC11 & hsa-mir-196a-2 & $v$ \\
\hline 5 & Loss & 22 & q13.1 & MKL1 & & $v$ \\
\hline 5 & Loss & 22 & q12.1 & CHEK2 & & 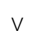 \\
\hline
\end{tabular}

The column "Number of samples" represents the number of patients with a particular genomic abnormality. For each variation the type of variation (gain or loss), location on chromosome and band, the gene located in this locus and miRNAs located in this locus are enlisted. Variants reported in the Database of Genomic Variants (DGV) database in genes are denoted as " $\mathrm{v}$ " and if none reported as "-" under the column DGV

on a thermocycler, and counted for fluorescent signal. Absolute copy numbers of target and reference sequences were determined by Poisson algorithms [8]. The RNase P (Ribonuclease P) amplicon maps within the single exon RPPH1 gene on 14q11.2 was used as the standard reference assay for copy number analysis [11]. Validation results were as follows: EGFR amplification was detected in 29 of 32 patient samples (Fig. 4), PTCH1 was amplified in 18 of 32 patients (Fig. 5) and CHEK2 was deleted in 27 of 32 (Fig. 6). Surprisingly, PTPN11 was deleted in $7 / 32$ whilst it was amplified in $17 / 32$ specimens (Fig. 7).

It is important to note that in result of normal cell admixture within tumor samples a high CNV means either high fraction of tumor cells with relatively high copy number in each cell or low fraction of tumor cells with very high copy number in each cell. Likewise, low CNV means either high fraction of tumor cells with relatively low copy number in each cell or low fraction of tumor cells with relatively very

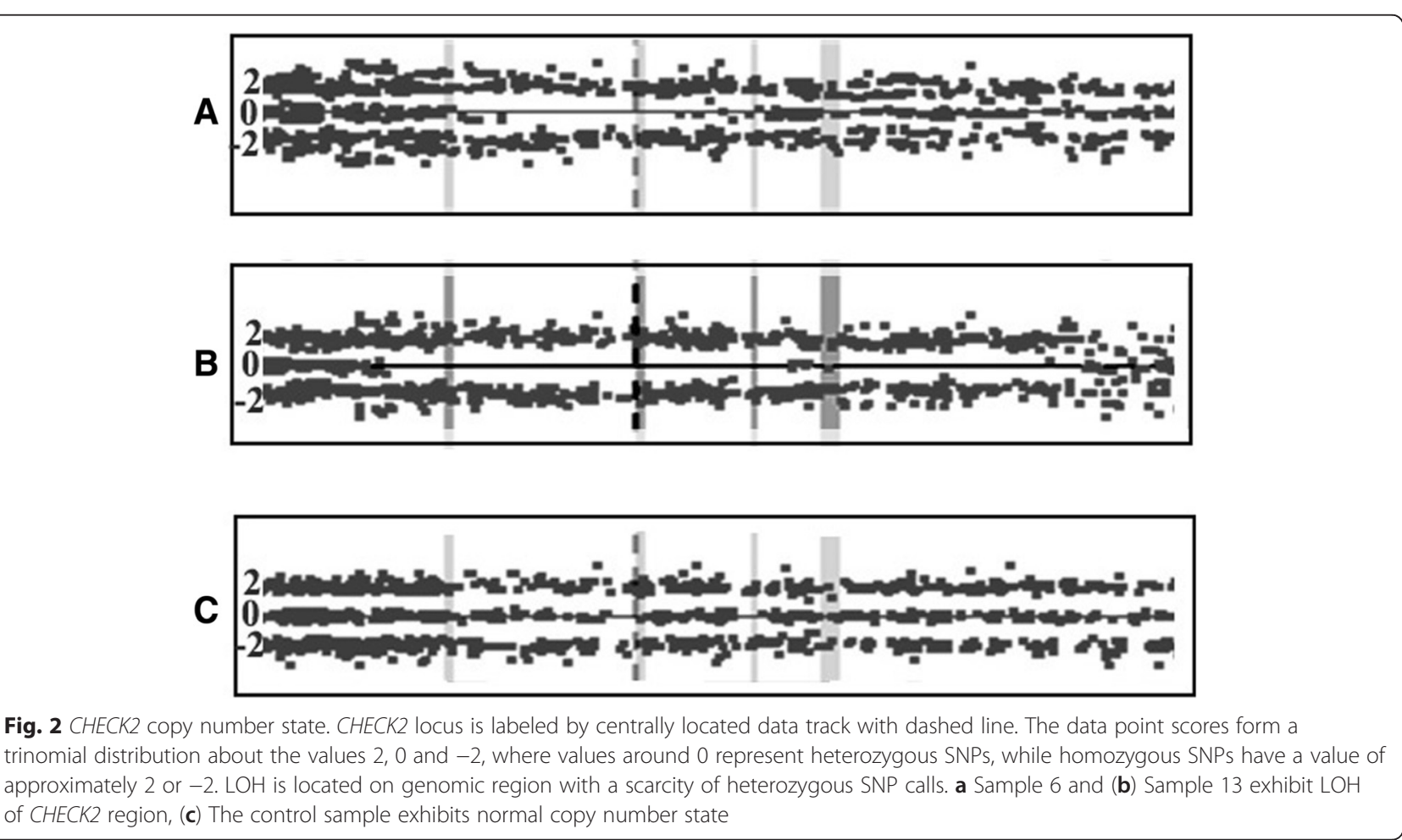



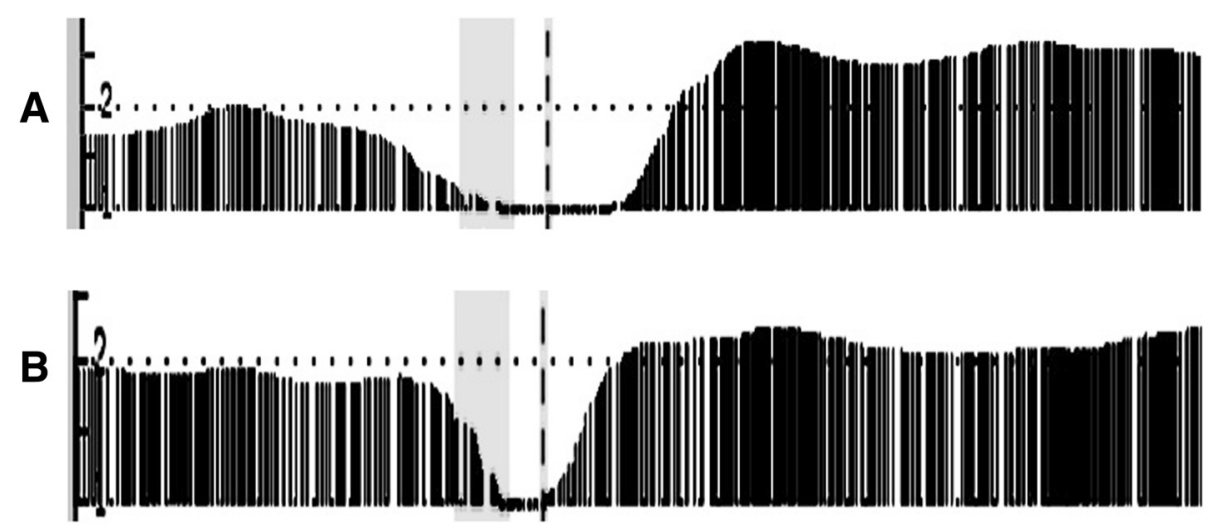

Fig. 3 VHL locus (Ch.3 p25.3) deletion data display. The smooth signal derived from the raw data is displayed. The smooth signal value is calculated using the intensity values of the flanking probes and the data is displayed by a continuous line which is filled down to the $x$ axis. Focusing on VHL locus was done by selecting the specific region of the chromosome and zooming in such that this region spans the entire $x-$ axis. The data points containing the probe values are overlaid on the graph. Smooth signal value can be between zero or four depending on the Log(2) ratio raw values of the surrounding probes. a Sample 7 and (b) Sample ten smooth signal is one exhibiting deletion in VHL locus (labeled with dashed line). Probes around the area are normal (around 2)

low copy number in each cell. In short if the fraction of tumor cells is low then the relative copy number (both high and low $\mathrm{CNV}$ ) is even higher than what is reported. In our cohort, histopathologic assessment by a pathologist determined that 47 percent of samples had more than $95 \%$ tumor, 7 percent had more than $90 \%$ tumor, 29 percent of samples had more than 70-80 \% tumor, 14 percent of samples had between $50-70 \%$ tumor and 3 percent had between $40-50 \%$ tumor.

\section{Discussion}

We report here for the first time a genome-wide, highresolution systematic analysis of chromosomal changes in hemangioblastoma. Using the SNP array 6 (Affymetrix) we analyzed 1.8 million genetic markers genome wide to identify amplifications/deletions up to single gene level.
We identified a total of $94 \mathrm{CNVs}, 23$ of them involved noncoding regions. 56 (31 gains and 25 losses) were found in at least two specimens. The most frequently gained regions were on chromosomes 1 (p36.32) and 7 (p11.2). The most frequently deleted region was on chromosome 12 (q24.13).

Our findings provide the first high-resolution genomewide view of chromosomal changes in hemangioblastoma and identify 23 common, ie found in 4 or more patients, candidate genes for hemangioblastoma pathogenesis (Table 3): EGFR, PRDM16, PTPN11, HOXD11, HOXD13, FLT3, PTCH, FGFR1, FOXP1, GPC3, HOXC13, HOXC11, MKL1, CHEK2, IRF4, GPHN, IKZF1, RB1, HOXA9, HOXA11 and several microRNA, including hsa-mir-196a2. We note that some of these microalterations have been reported very rarely previously in tissue samples from

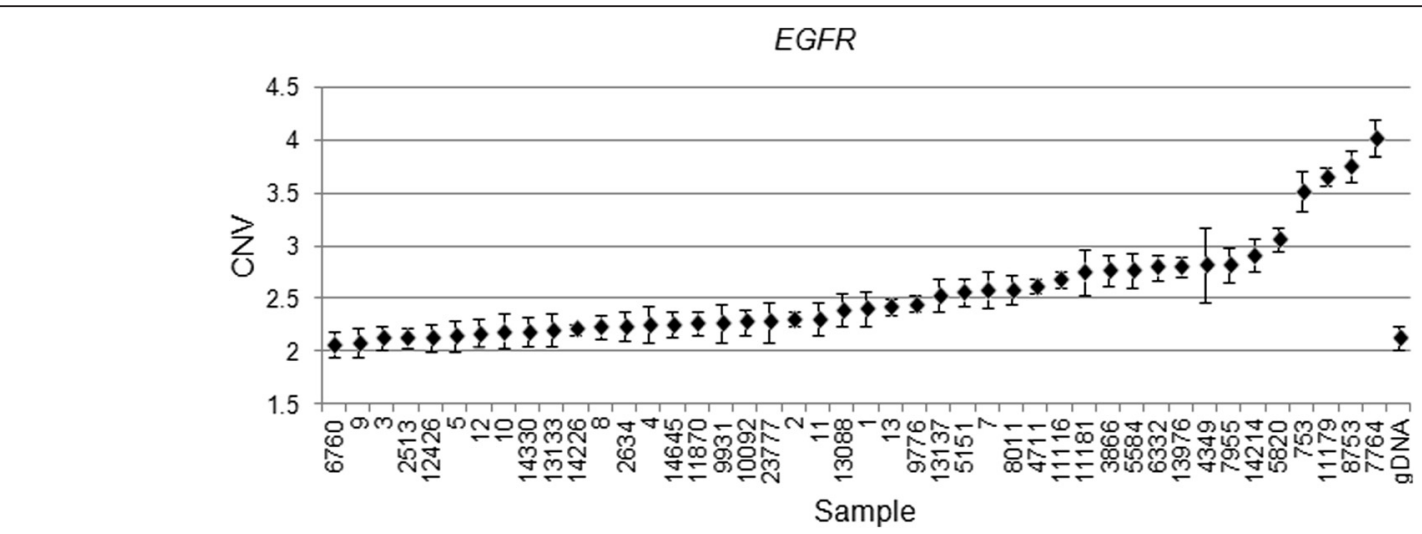

Fig. 4 EGFR Copy number variation (CNV) values for clinical samples and one normal genomic DNA sample. Genomic DNA copy number alterations were assessed via ddPCR. The CNV is shown as the number of copies and the Poisson distribution at $95 \%$ confidence interval. The copy number value of normal diploid sequence has a score of two. Copy number above two means amplification in that region and copy number below two means deletion in that region 
PTCH1

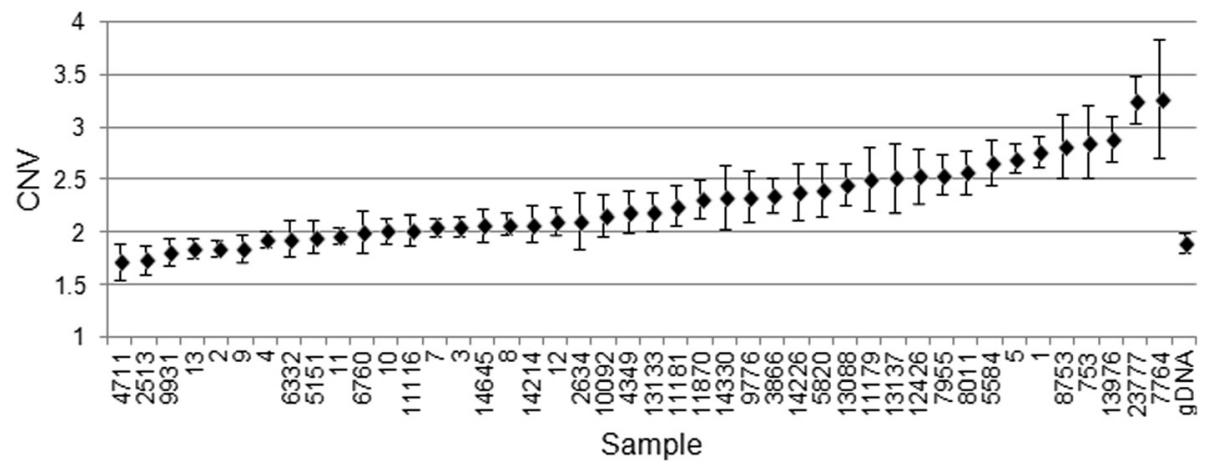

Fig. 5 PTCH1 Copy number variation (CNV) values for clinical samples and one normal genomic DNA sample. Genomic DNA copy number alterations were assessed via ddPCR. The CNV is shown as the number of copies and the Poisson distribution at $95 \%$ confidence interval. The copy number value of normal diploid sequence has a score of two. Copy number above two means amplification in that region and copy number below two means deletion in that region

healthy subjects (according to Database of Genome Variants (DGV), which are reported in Table 3. However, in our tumor samples these alterations are significantly more common $(\mathrm{p}<0.00001)$.

Functional annotation analysis by David [12] reviled that two pathways are prominently affected in the hemangioblastoma samples: the cell proliferation and angiogenesis promoting pathways. The cell proliferation pathway includes the following genes: CHEK2, EGFR, FGFR, FLT3 and PTCH1. The angiogenesis pathway includes the genes $E G F R$ and FGFR, which are significant because they are also involved in blood vessel formation. Importantly, three of these genes were verified by ddPCR: EGFR, CHEK2 and $P T C H 1$. Furthermore, PTPN11 was selected for verification as it was lost strikingly often (Table 3 ).

Epidermal growth factor receptor $(E G F R)$ is a tyrosine kinase receptor that has been documented with increased expression in a variety of human cancers such as breast cancer, [13], early stage non-small-cell lung cancers and gliomas $[14,15]$. Our findings are in line with three prior immunohistochemical studies that found EGFR overexpression in hemangioblastomas [16] [17] [18]. Fibroblast growth factor receptor 1 (FGFR1) micro deletions have been reported in myeloid and lymphoid neoplasms [19]. Interestingly we discovered that the FGFR1 deletion involves the FGFR1 2nd intron (chromosome 8, 38291333-38314367), which according to UCSC genome browser hg19 includes a regulatory site. Checkpoint kinase 2 (CHEK2) has been implicated in DNA repair, cell cycle arrest, and apoptosis in response to DNA double-strand breaks [20]. Mutations in CHEK2 have been reported to be possibly associated with breast cancer and liposarcoma development [21, 22]. The protein patched homolog 1 (PTCH1) is a sonic hedgehog receptor. Loss of function mutations in $\mathrm{PTCH} 1$ are associated with development of various types of cancers, including medulloblastoma [23, 24], pancreatic cancer [25] and

\section{Check2}

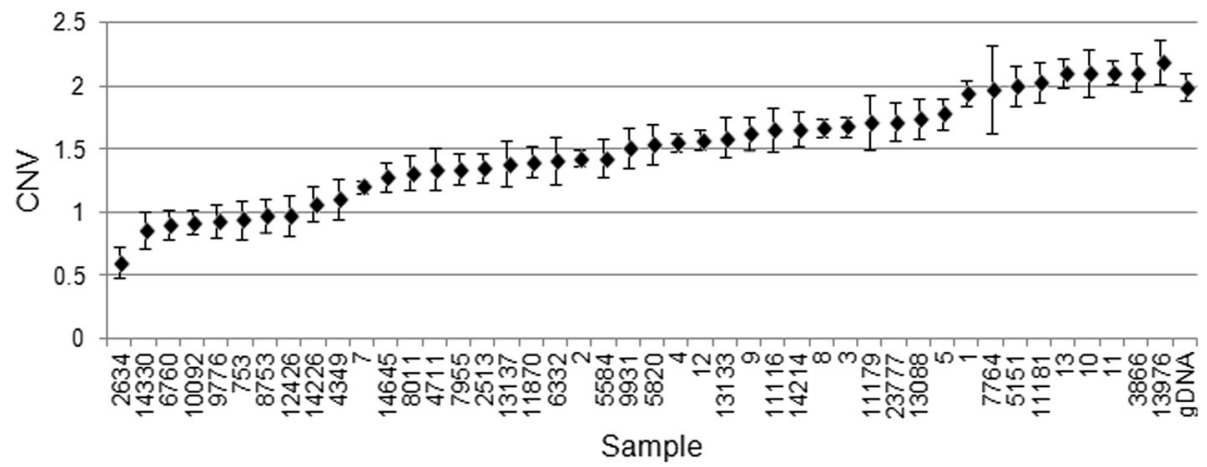

Fig. 6 CHEK2 Copy number variation (CNV) values for clinical samples and one normal genomic DNA sample. Genomic DNA copy number alterations were assessed via ddPCR. The CNV is shown as the number of copies and the Poisson distribution at $95 \%$ confidence interval. The copy number value of normal diploid sequence has a score of two. Copy number above two means amplification in that region and copy number below two means deletion in that region 
PTPN11

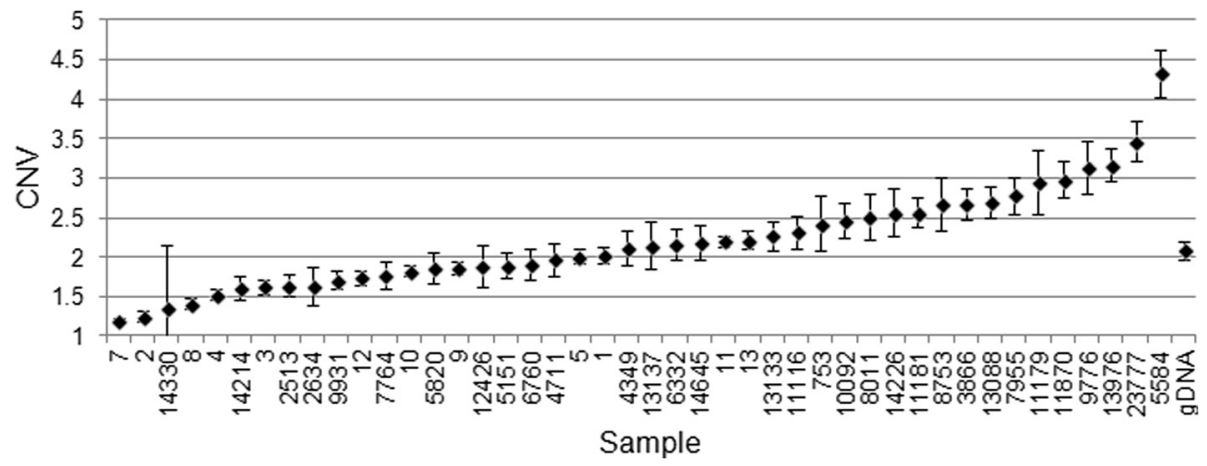

Fig. 7 PTPN11 Copy number variation (CNV) values for clinical samples and one normal genomic DNA sample. Genomic DNA copy number alterations were assessed via ddPCR. The CNV is shown as the number of copies and the Poisson distribution at $95 \%$ confidence interval. The copy number value of normal diploid sequence has a score of two. Copy number above two means amplification in that region and copy number below two means deletion in that region

colorectal cancer [26]. In contrast to these observations, we find consistent PTCH1 gains in our cohort of hemangioblastoma patients (Table 3 ). In support of our findings there are reports of PTCH1 also acting as an oncogene in a mouse model of skin basal cell carcinomas [27].

FMS-like tyrosine kinase 3 (FLT3) plays a role in hematopoiesis including early hematologic differentiation and early B and T-cell development [28] and dendritic cells differentiation [29]. Activating FLT3 mutations are some of the most common molecular abnormalities in acute myeloid leukemia (AML) [30]. Interestingly, in our data, we find losses of FLT3 (Table 3). Consistent with our findings, others have reported deletions in FLT3 in AML as well [31] [32]. Among the altered genes mapped in many of the recurrently gained regions we recognized several HOX genes (Table 3) and microRNAs (miRNAs) residing in the same region. $H O X$ genes encode master transcription factors important in development and have been reported to be commonly altered in human solid tumors [33]. On the other hand, miRNAs are small regulatory RNAs that have recently been implicated in a variety of cancers [34]. miR-196a-2 and miR-196b gain were the most common miRNA CNV in our patients. Interestingly, miR-196a-2 differs from miR-196b by one nucleotide [35]. The miR-196 gene family is located in the regions of homeobox $(H O X)$ transcription factors that are essential for embryogenesis. Up-regulation of miR-196a has been found in breast cancer, adenocarcinoma, leukemia and esophageal adenocarcinoma [35]. Accordingly the relevant causative change may actually be altered miRNA expression rather than $H O X$ gene expression. Other studies have reported a 12 -fold increase in miR-9 and a 15-fold decrease of miR-200a in hemangioblastomas distinguishing hemangioblastomas from metastatic clear cell renal cell carcinomas in the CNS [36]. Prdm16 is preferentially expressed by stem cells throughout the nervous and haematopoietic systems and promotes stem cell maintenance [37]. Megakaryoblastic leukemia protein-1 (MKL1), is a transcription factor that regulates many processes, including remodeling of neuronal networks and epithelialmesenchymal transition [38]. Moreover, deregulation by genetic alterations and/or altered MKL1 transcription has been shown to have role in myeloproliferative neoplasms [39]. Finally and most interestingly, protein tyrosine phosphatase, non-receptor type 11 (PTPN11) gene encodes the tyrosine phosphatase SHP2 protein required for RTK signaling and has a role in survival, proliferation and differentiation [40]. The fact that we find the PTPN11 gene is deleted in some hemangioblastoma patients and is amplified in others may suggest that these tumors actually originate from different cellular lineages. In fact, we found that spinal tumors were overwhelmingly deleted in the PTPN11 region: 5 of 7 spinal tumors were deleted whilst 1 was amplified and 1 was chromosomally normal at this locus. In contrast, cerebellar hemangioblastomas were generally amplified: 16 out 37 were indeed amplified. However, 10 cerebellar tumors were deleted and 11 of 37 were normal in this genomic region. Interestingly, differential overexpression or deletion of PTPN11 has been shown in other tumors. For example, mutations in PTPN11 has been reported to be associated with development of Juvenile Myelomonocytic Leukemia (JMML) [41], acute myeloblastic leukemia (AML) [42], and acute lymphoblastic leukemia (ALL) [43]. Overexpression in gastric carcinomas has been reported [44]. In contrast, PTPN11 has a tumor-suppressor function in liver [45] and cartilage [46]. Accordingly, decreased PTPN11 expression was detected in a subfraction of human hepatocellular carcinoma specimens [45]. Thus, in contrast to its common pro-oncogenic role in hematopoietic and epithelial cells, PTPN11 may act as a tumor suppressor in cartilage. Accordingly, we hypothesize that the PTPN11 gene may 
act in a cell-specific manner: as a tumor suppressor on one hand in the progenitor cells of spinal hemangioblastomas, whilst it acts as an oncogene in the cells of origin of cerebellar hemangioblastoma tumors.

Previous analysis of six VHL-related CNS hemangioblastomas showed loss of chromosome $3 p$ or the whole of chromosome 3 to be the most common abnormality, which is detected in $70 \%$ and loss of 1p11-p31 in $10 \%$ [47]. More relevant to our findings, published CGH studies on 10 sporadic cerebellar hemangioblastomas detected losses of chromosomes 3 (70 \%), 6 (50\%), 9 (30\%), and $18 \mathrm{q}(30 \%)$ and a gain of chromosome 19 (30 \%) [48]. We indeed detected losses and gains in these areas but they were not frequent (15-20\%). Chromosome 3 losses were more abundant, mostly on p13 (5 samples FOXP1) and p25 (3 samples showed PPARG loss and 2 samples showed VHL loss). Interestingly, FOXP1 transcription factor, located on chromosome 3(p13), can function as a tumor suppressor gene. Low expression in glioma [49] and Hodgkin lymphoma has been shown [50]. Differences in methodology, sample size and definition of aberration inclusion criteria may account for some of the apparent inconsistencies between previous studies and our findings. For example, previous results were obtained from VHL-related hemangioblastomas using techniques that identify deletions that are larger than $2 \mathrm{Mb}$. In the current study we used modern CGH microarrays which scan the DNA every $1 \mathrm{~kb}$ and thus we were able to identify very subtle genomic changes. One of the most striking observations in our study is that many CNVs affected single genes (Table 3) and revealed candidate genes, which have not been implicated in hemangioblastomas. This represents an important outcome of this study compared with previous investigations using CGH. Some of the new genes identified here as affected by CNVs in hemangioblastoma may serve as targets for future precisely targeted anti- cancer therapy. For example, antiangiogenic therapy can be given to patients with lesions that are not resectable.

\section{Conclusions}

In this study, we have demonstrated in two different tumor cohorts and using two different techniques for copy number alteration detection, SNP and digital PCR, that Chek2 is deleted and EGFR, PTPN11, Ptch1 amplified in majority of hemangioblastoma patients. EGFR is the only gene that has been previously reported as a candidate gene with hemangioblastoma. Independent of HB tumor location PTPN11 may act as tumor suppressor or oncogene depending on the tumor cell of origin. These findings have potentially relevant clinical value, as this the first high resolution for chromosomal alteration in HB. Future research should be dedicated to the prospective validation of these alterations and further characterization of tumors that carry the deletions/amplifications, as well as of defining the role of these genes. This may offer insights into hemangioblastoma biology, provide DNA-based markers that can be analyzed by FISH suitable for routine clinical applications and eventually lead to the development of effective targeted therapies for HB.

\begin{abstract}
Abbreviations
CNVs: Copy number variations; HB: Hemangioblastomas; CNS: Central nervous system; VHL: von Hippel-Lindau; CGH: Comparative genomic hybridization; SNP: Single nucleotide polymorphism; LOH: Loss of heterozygosity; ddPCR: Droplet Digital PCR; CNV: Copy number variation; FFPE: Formalin Fixed Paraffin Embedded; RNase P: Ribonuclease P; EGFR: Epidermal growth factor receptor; FGFR: Fibroblast growth factor receptor 1; CHEK2: Checkpoint kinase 2; PTCH1: Protein patched homolog 1; FLT3: FMS-like tyrosine kinase 3; AML: Acute myeloid leukemia; miRNA: microRNA; MKL1: Megakaryoblastic leukemia protein-1; PTPN11: protein tyrosine phosphatase non-receptor type 11.
\end{abstract}

\section{Competing interests}

The authors declare that they have no competing interest.

\section{Authors' contributions}

RMS conceived, designed and coordinated the study, performed the $\mathrm{CGH}$ analysis and drafted the manuscript, IM carried out the droplet digital PCR experiments, IB and DN participated in collecting the FFPE samples, JJ and CD carried out the CGH experiments, JR provided advice and revised the manuscript, MY, SC and AT participated in collecting the frozen samples, designing and coordinating of the study. All authors read and approved the final manuscript.

\section{Acknowledgments}

This research was funded by The Sheba Medical Research fund. We thank Sarah South, PhD, University of Utah, for insightful remarks on Affymetrix $\mathrm{CGH}$ analysis.

\section{Author details}

${ }^{1}$ Pediatric Hemato-Oncology, Edmond and Lilly Safra Children's Hospital and Cancer Research Center, Sheba Medical Center, Tel Hashomer affiliated to the Sackler School of Medicine, Tel-Aviv University, Tel Aviv, Israel. ${ }^{2}$ Sackler Faculty of Medicine, Tel Aviv University, Tel Aviv, Israel. ${ }^{3}$ Institute of Pathology, Sheba Medical Center, Tel Hashomer, Israel. ${ }^{4}$ Division of Tropical Health and Medicine, James Cook University, Townsville, QLD, Australia.

${ }^{5}$ Department of Pediatric Neurosurgery, Dana Children's Hospital,

Tel-Aviv-Sourasky Medical Center, Tel-Aviv, Israel.

Received: 19 June 2015 Accepted: 6 January 2016

Published online: 14 January 2016

\section{References}

1. Lonser RR, Glenn GM, Walther M, Chew EY, Libutti SK, Linehan WM, et al. von Hippel-Lindau disease. Lancet. 2003;361(9374):2059-67. doi:10.1016/S0140-6736(03)13643-4.

2. Maher ER, Iselius L, Yates JR, Littler M, Benjamin C, Harris R, et al. Von Hippel-Lindau disease: a genetic study. J Med Genet. 1991;28(7):443-7.

3. McNeill A, Rattenberry E, Barber R, Killick P, MacDonald F, Maher ER. Genotype-phenotype correlations in VHL exon deletions. Am J Med Genet A. 2009;149A(10):2147-51.

4. Kaelin Jr WG. The von Hippel-Lindau tumour suppressor protein: $\mathrm{O} 2$ sensing and cancer. Nat Rev Cancer. 2008;8(11):865-73.

5. Ricketts C, Zeegers MP, Lubinski J, Maher ER. Analysis of germline variants in CDH1, IGFBP3, MMP1, MMP3, STK15 and VEGF in familial and sporadic renal cell carcinoma. PLoS ONE. 2009;4(6):e6037. doi:10.1371/journal.pone. 0006037.

6. Glasker S, Bender BU, Apel TW, van Velthoven V, Mulligan LM, Zentner J, et al. Reconsideration of biallelic inactivation of the VHL tumour suppressor gene in hemangioblastomas of the central nervous system. J Neurol Neurosurg Psychiatry. 2001;70(5):644-8. 
7. Dutt A, Beroukhim R. Single nucleotide polymorphism array analysis of cancer. Curr Opin Oncol. 2007;19(1):43-9. doi:10.1097/CCO. 0b013e328011a8c1.

8. Hindson BJ, Ness KD, Masquelier DA, Belgrader P, Heredia NJ, Makarewicz AJ, et al. High-throughput droplet digital PCR system for absolute quantitation of DNA copy number. Anal Chem. 2011;83(22):8604-10. doi:10.1021/ac202028g.

9. Hoebeeck J, van der Luijt R, Poppe B, De Smet E, Yigit N, Claes K et al. Rapid detection of $\mathrm{VHL}$ exon deletions using real-time quantitative PCR. Laboratory investigation; a journal of technical methods and pathology. 2005;85(1):24-33. doi:10.1038/labinvest.3700209.

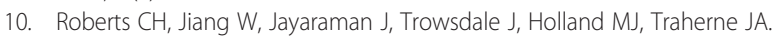
Killer-cell Immunoglobulin-like Receptor gene linkage and copy number variation analysis by droplet digital PCR. Genome Med. 2014;6(3):20. doi:10.1186/gm537.

11. Fernandez-Jimenez N, Castellanos-Rubio A, Plaza-Izurieta L, Gutierrez G, Irastorza I, Castano L, et al. Accuracy in copy number calling by qPCR and PRT: a matter of DNA. PLOS ONE. 2011;6(12):e28910. doi:10.1371/journal. pone.0028910.

12. da Huang W, Sherman BT, Lempicki RA. Bioinformatics enrichment tools: paths toward the comprehensive functional analysis of large gene lists. Nucleic Acids Res. 2009;37(1):1-13. doi:10.1093/nar/gkn923.

13. Shien T, Tashiro T, Omatsu M, Masuda T, Furuta K, Sato N, et al. Frequent overexpression of epidermal growth factor receptor (EGFR) in mammary high grade ductal carcinomas with myoepithelial differentiation. J Clin Pathol. 2005;58(12):1299-304. doi:10.1136/jcp.2005.026096.

14. Veale D, Kerr N, Gibson GJ, Kelly PJ, Harris AL. The relationship of quantitative epidermal growth factor receptor expression in non-small cell lung cancer to long term survival. Br J Cancer. 1993;68(1):162-5.

15. Rich JN, Hans C, Jones B, Iversen ES, McLendon RE, Rasheed BK, et al. Gene expression profiling and genetic markers in glioblastoma survival. Cancer Res. 2005;65(10):4051-8. doi:10.1158/0008-5472.CAN-04-3936.

16. Chen GJ, Karajannis MA, Newcomb EW, Zagzag D. Overexpression and activation of epidermal growth factor receptor in hemangioblastomas. Neurooncol. 2010;99(2):195-200. doi:10.1007/s11060-010-0125-9.

17. Bohling T, Hatva E, Kujala M, Claesson-Welsh L, Alitalo K, Haltia M. Expression of growth factors and growth factor receptors in capillary hemangioblastoma. J Neuropathol Exp Neurol. 1996;55(5):522-7.

18. Reifenberger $G$, Reifenberger J, Bilzer $T$, Wechsler W, Collins VP. Coexpression of transforming growth factor-alpha and epidermal growth factor receptor in capillary hemangioblastomas of the central nervous system. Am J Pathol. 1995;147(2):245-50.

19. Yang JJ, Park TS, Choi JR, Park SJ, Cho SY, Jun KR, et al. Submicroscopic deletion of FGFR1 gene is recurrently detected in myeloid and lymphoid neoplasms associated with ZMYM2-FGFR1 rearrangements: a case study. Acta Haematol. 2012;127(2):119-23. doi:10.1159/000334707.

20. Jackson SP, Bartek J. The DNA-damage response in human biology and disease. Nature. 2009:461(7267):1071-8. doi:10.1038/nature08467.

21. Walsh T, Casadei S, Coats KH, Swisher E, Stray SM, Higgins J, et al. Spectrum of mutations in BRCA1, BRCA2, CHEK2, and TP53 in families at high risk of breast cancer. JAMA. 2006;295(12):1379-88. doi:10.1001/jama.295.12.1379.

22. Manoukian S, Peissel B, Frigerio S, Lecis D, Bartkova J, Roversi G, et al. Two new CHEK2 germ-line variants detected in breast cancer/sarcoma families negative for BRCA1, BRCA2, and TP53 gene mutations. Breast Cancer Res Treat. 2011;130(1):207-15. doi:10.1007/s10549-011-1548-5.

23. Kool M, Jones DT, Jager N, Northcott PA, Pugh TJ, Hovestadt V, et al. Genome Sequencing of SHH Medulloblastoma Predicts Genotype-Related Response to Smoothened Inhibition. Cancer Cell. 2014;25(3):393-405. doi:10.1016/j.ccr.2014.02.004

24. Mascaro Cordeiro B, Dias Oliveira I, de Seixas Alves MT, Saba-Silva N, Capellano AM, Cavalheiro S et al. SHH, WNT, and NOTCH pathways in medulloblastoma: when cancer stem cells maintain self-renewal and differentiation properties. Childs Nerv Syst. 2014. doi:10.1007/s00381-014-2403-x

25. Xin Y, Shen XD, Cheng L, Hong DF, Chen B. Perifosine inhibits S6K1-Gli1 signaling and enhances gemcitabine-induced anti-pancreatic cancer efficiency. Cancer Chemother Pharmacol. 2014;73(4):711-9. doi:10.1007/s00280-014-2397-9.

26. Chung JH, Bunz F. A loss-of-function mutation in PTCH1 suggests a role for autocrine hedgehog signaling in colorectal tumorigenesis. Oncotarget. 2013;4(12):2208-11.
27. Kang HC, Wakabayashi Y, Jen KY, Mao JH, Zoumpourlis V, Del Rosario R, et al. Ptch1 overexpression drives skin carcinogenesis and developmental defects in K14Ptch(FVB) mice. J Invest Dermatol. 2013;133(5):1311-20. doi:10.1038/jid.2012.419.

28. Schmidt-Arras D, Schwable J, Bohmer FD, Serve H. Flt3 receptor tyrosine kinase as a drug target in leukemia. Curr Pharm Des. 2004;10(16):1867-83.

29. Laouar Y, Welte T, Fu XY, Flavell RA. STAT3 is required for Flt3L-dependent dendritic cell differentiation. Immunity. 2003;19(6):903-12.

30. Moreno I, Martin G, Bolufer P, Barragan E, Rueda E, Roman J, et al. Incidence and prognostic value of FLT3 internal tandem duplication and D835 mutations in acute myeloid leukemia. Haematologica. 2003;88(1):19-24.

31. Armstrong SA, Mabon ME, Silverman LB, Li A, Gribben JG, Fox EA, et al. FLT3 mutations in childhood acute lymphoblastic leukemia. Blood. 2004;103(9): 3544-6. doi:10.1182/blood-2003-07-2441.

32. Chang $P$, Kang M, Xiao A, Chang J, Feusner J, Buffler $P$, et al. FLT3 mutation incidence and timing of origin in a population case series of pediatric leukemia. BMC Cancer. 2010;10:513. doi:10.1186/1471-2407-10-513.

33. Bhatlekar S, Fields JZ, Boman BM. HOX genes and their role in the development of human cancers. J Mol Med (Berl). 2014;92(8):811-23. doi:10.1007/s00109-014-1181-y.

34. Di Leva G, Garofalo M, Croce CM. MicroRNAs in cancer. Annu Rev Pathol. 2014;9:287-314. doi:10.1146/annurev-pathol-012513-104715.

35. Chen C, Zhang Y, Zhang L, Weakley SM, Yao Q. MicroRNA-196: critical roles and clinical applications in development and cancer. J Cell Mol Med. 2011; 15(1):14-23. doi:10.1111/j.1582-4934.2010.01219.x.

36. Venneti S, Boateng LA, Friedman JR, Baldwin DA, Tobias JW, Judkins AR, et al. MiRNA-9 and MiRNA-200a distinguish hemangioblastomas from metastatic clear cell renal cell carcinomas in the CNS. Brain Pathol. 2012 22(4):522-9. doi:10.1111/j.1750-3639.2011.00551.x.

37. Chuikov S, Levi BP, Smith ML, Morrison SJ. Prdm16 promotes stem cell maintenance in multiple tissues, partly by regulating oxidative stress. Nat Cell Biol. 2010:12(10):999-1006. doi:10.1038/ncb2101.

38. Tijssen MR, Ghevaert C. Transcription factors in late megakaryopoiesis and related platelet disorders. J Thromb Haemost. 2013:11(4):593-604. doi:10. 1111/jth.12131.

39. Scharenberg MA, Chiquet-Ehrismann R, Asparuhova MB. Megakaryoblastic leukemia protein-1 (MKL1): Increasing evidence for an involvement in cancer progression and metastasis. Int J Biochem Cell Biol. 2010;42(12): 1911-4. doi:10.1016/j.biocel.2010.08.014.

40. Agazie YM, Movilla N, Ischenko I, Hayman MJ. The phosphotyrosine phosphatase SHP2 is a critical mediator of transformation induced by the oncogenic fibroblast growth factor receptor 3. Oncogene. 2003; 22(44):6909-18. doi:10.1038/sj.onc.1206798.

41. Tartaglia M, Niemeyer CM, Fragale A, Song X, Buechner J, Jung A, et al. Somatic mutations in PTPN11 in juvenile myelomonocytic leukemia, myelodysplastic syndromes and acute myeloid leukemia. Nat Genet. 2003; 34(2):148-50. doi:10.1038/ng1156

42. Ucar C, Calyskan U, Martini S, Heinritz W. Acute myelomonocytic leukemia in a boy with LEOPARD syndrome (PTPN11 gene mutation positive). J Pediatr Hematol Oncol. 2006;28(3):123-5. doi:10.1097/01.mph.0000199590. 21797.0b.

43. Tartaglia M, Martinelli S, Cazzaniga G, Cordeddu V, lavarone I, Spinelli $\mathrm{M}$, et al. Genetic evidence for lineage-related and differentiation stagerelated contribution of somatic PTPN11 mutations to leukemogenesis in childhood acute leukemia. Blood. 2004;104(2):307-13. doi:10.1182/blood-2003-11-3876.

44. Kim JS, Shin OR, Kim HK, Cho YS, An CH, Lim KW, et al. Overexpression of protein phosphatase non-receptor type 11 (PTPN11) in gastric carcinomas. Dig Dis Sci. 2010;55(6):1565-9. doi:10.1007/s10620-009-0924-z.

45. Bard-Chapeau EA, Li S, Ding J, Zhang SS, Zhu HH, Princen F, et al. Ptpn11/ Shp2 acts as a tumor suppressor in hepatocellular carcinogenesis. Cancer Cell. 2011;19(5):629-39. doi:10.1016/j.ccr.2011.03.023.

46. Yang W, Wang J, Moore DC, Liang H, Dooner M, Wu Q, et al. Ptpn11 deletion in a novel progenitor causes metachondromatosis by inducing hedgehog signalling. Nature. 2013;499(7459):491-5. doi:10.1038/ nature12396.

47. Lui WO, Chen J, Glasker S, Bender BU, Madura C, Khoo SK, et al. Selective loss of chromosome 11 in pheochromocytomas associated with the $\mathrm{VHL}$ syndrome. Oncogene. 2002;21(7):1117-22. doi:10.1038/sj.onc.1205149.

48. Sprenger SH, Gijtenbeek JM, Wesseling P, Sciot R, van Calenbergh F, Lammens $\mathrm{M}$, et al. Characteristic chromosomal aberrations in sporadic 
cerebellar hemangioblastomas revealed by comparative genomic hybridization. J Neuro-Oncol. 2001;52(3):241-7.

49. Xue L, Yue S, Zhang J. FOXP1 has a low expression in human gliomas and its overexpression inhibits proliferation, invasion and migration of human glioma U251 cells. Mol Med Rep. 2014;10(1):467-72. doi:10.3892/mmr.2014.2197.

50. Nagel S, Meyer C, Kaufmann M, Drexler HG, MacLeod RA. Deregulated FOX genes in Hodgkin lymphoma. Genes Chromosomes Cancer. 2014;53(11): 917-33. doi:10.1002/gcc.22204

Submit your next manuscript to BioMed Central and we will help you at every step:

- We accept pre-submission inquiries

- Our selector tool helps you to find the most relevant journal

- We provide round the clock customer support

- Convenient online submission

- Thorough peer review

- Inclusion in PubMed and all major indexing services

- Maximum visibility for your research

Submit your manuscript at www.biomedcentral.com/submit 